\title{
Cortical Perfusion and Gray Matter Weight in Frontal Lobe Dementia
}

\author{
Gene E. Alexander, Ph.D. \\ Isak Prohovnik, Ph.D. \\ Harold A. Sackeim, Ph.D. \\ Yaakov Stern, Ph.D. \\ Richard Mayeux, M.D.
}

To evaluate the pathophysiology of frontal lobe dementia (FLD), the authors compared regional cerebral blood flow ( $r C B F$ ) in matched groups of FLD, probable Alzheimer's disease $(A D)$, and major depression patients and normal control subjects $(\mathrm{n}=$ 7 each). The planar xenon-133 technique allowed full quantification of cortical perfusion and estimates of the relative weight of gray matter (wg). FLD patients showed lower blood flow and wg in the frontal cortex than the other groups. Mean cortical perfusion was correlated with cortical wg in the FLD group only. These findings 1) suggest that matched reductions of frontal gray matter weight and perfusion occur in FLD and 2) support the use of $r C B F$ in distinguishing FLD from $A D$ and severe depression.

(The Journal of Neuropsychiatry and Clinical Neurosciences 1995; 7:188-196)

$\mathrm{P}$ rogressive neurodegenerative diseases that principally affect the anterior cerebral cortex have, in recent years, been ascribed to the category of frontal lobe-type dementia (FLD). ${ }^{1,2}$ Although several histopathological variants have been reported among patients diagnosed with FLD, ${ }^{3-7}$ lobar atrophy of the frontotemporal cortex in the absence of Alzheimer's pathology has been viewed as the most salient neuropathological feature. Structural imaging studies of FLD sometimes reveal focal atrophy involving the frontotemporal regions, ${ }^{5,8}$ but CT and MRI often show only diffuse atrophy. ${ }^{9,10}$

In contrast, cortical perfusion appears to be consistently altered among FLD patients, with prominent blood flow deficits in the anterior cortex. ${ }^{11-15}$ This pattern of hypoperfusion has been distinguished from those in patients with Alzheimer's disease (AD) and vascular dementia, as well as healthy control subjects. ${ }^{12,13,15}$ The specificity of this finding to FLD is unknown with respect to the frontal hypoperfusion and decreased metabolism reported among severely depressed patients. ${ }^{16-20}$

Further, morphometric examination of brain tissue from patients diagnosed with the Pick's disease variant of FLD has shown greater reductions in brain weight and increased cortical thinning of the frontotemporal regions in these patients compared with AD patients and agematched normal control subjects. ${ }^{21}$ This difference in gray matter weight $(\mathrm{wg})$ has not yet been studied by imaging methods. The index of gray matter weight obtained by xenon-133 regional cerebral blood flow (rCBF)

Received May 18, 1994; revised August 25, 1994; accepted August 26, 1994. From the Departments of Brain Imaging and Biological Psychiatry, New York State Psychiatric Institute; and the Departments of Neurology, Psychiatry, and Radiology, College of Physicians and Surgeons, Columbia University, New York. Address correspondence to Dr. Prohovnik, Department of Brain Imaging, New York State Psychiatric Institute, Unit \#72, 722 West 168th Street, New York, NY 10032.

Copyright @ 1995 American Psychiatric Press, Inc. 
is an inferential measure of gray matter relative to wholebrain perfused tissue and is correlated with weight of gray matter in the normal brain. ${ }^{22,23}$ Reductions in relative wg have been associated with dementia severity and may be indicative of increased atrophy or cell loss in the cerebral cortex. $^{23}$

We hypothesized that if FLD is prominently characterized by dysfunction and atrophy of frontotemporal cortex, then anterior cortical regions would show reductions in perfusion and gray matter weight in this type of dementia.

To test these hypotheses, 7 patients with clinical diagnoses of FLD or Pick's disease were compared with groups of $\mathrm{AD}$, depressed, and normal control subjects by using xenon-133 rCBF. All groups were matched in demographic characteristics, and the two dementia groups had similar levels of clinical dementia severity. The groups were compared on indices of cortical perfusion and gray matter weight to investigate the pathophysiological characteristics of FLD that are distinguishable from those of $\mathrm{AD}$, depression, and normal aging. The two dementia samples included patients in the early stages of dementia (that is, with short illness durations), allowing us to test the sensitivity of our imaging methods. For the same reason, we included patients with severe depression as a comparison group.

\section{METHODS}

\section{Subjects}

Seven patients with a clinical diagnosis of possible/probable Pick's disease or FLD were referred for clinical evaluations of cerebral blood flow. Patients meeting NINCDS-ADRDA ${ }^{24}$ criteria for probable Alzheimer's disease (participants in a longitudinal study of $\mathrm{rCBF}$ in $\mathrm{AD}$ ), were matched to the FLD group on dementia severity by using a modified version of the Folstein Mini-Mental State Examination ${ }^{25}\left(\mathrm{mMMS}^{26}\right)$ and estimates of illness duration. The $\mathrm{AD}$ group $(n=7)$ was also matched to the FLD patients on distributions of age, education, and gender.

A group of elderly patients with major depression (MDD, $n=7)$ and a normal control group $(n=7)$ were matched to the FLD group on the same demographic characteristics. The depressed patients met research criteria for endogenous major depressive disorder when assessed with the Schedule for Affective Disorders and Schizophrenia, ${ }^{27}$ had a mean Hamilton Depression ${ }^{28}$ score of 38.7 (range 34-44); and had no history of neurological illness or injury. The normal control subjects were healthy volunteers with no history of neurological or psychiatric illness. For all groups, there was also no history of drug or alcohol abuse in the past year and no concurrent medical illness that could influence $\mathrm{rCBF}$ measurement. The AD patients and normal control subjects were part of the sample described by Prohovnik et al., ${ }^{29}$ and the depressed patients were included in the series reported by Sackeim et al. ${ }^{17}$ The test procedures were fully explained to all subjects, and informed consent was obtained.

Patients included in the FLD group were referred for rCBF from a clinic for memory disorders, where they received a comprehensive dementia evaluation, including a neurological examination, neuropsychological testing, laboratory tests, and CT or MRI scans. Only patients who received a diagnosis of possible/probable Pick's disease or FLD based on clinical evaluations prior to rCBF measurement were included in the FLD group. Clinical and behavioral symptoms of patients receiving the diagnosis of FLD included dementia with prominent personality change, disinhibition, oral/dietary hyperactivity, mutism, and echolalia. None of the patients had a clinical history of stroke, head injury, or other neurological illness, and none showed evidence of significant cerebrovascular disease on structural neuroimaging. Three of the 7 FLD patients revealed focal atrophy on MRI or $\mathrm{CT}$, involving the frontal or anterior temporal regions. The remaining 4 FLD patients either showed diffuse atrophy or were within normal limits.

Patients in the AD group underwent the same clinical evaluation as the FLD patients. Medical examinations and laboratory screening tests for the depressed and normal subjects were also obtained. The depressed patients were free of psychotropic medications for at least 5 days before the $\mathrm{rCBF}$ measurement (range 6-30 days) with the exception of low-dose lorazepam (up to $3 \mathrm{mg} /$ day, but not within 10 hours of the rCBF assessment). One FLD patient received thioridazine hydrochloride $(50 \mathrm{mg}$ po qid) and triazolam $(0.5 \mathrm{mg}$ po hs) daily. No other patients or normal control subjects received medication at the time of $\mathrm{rCBF}$ testing.

\section{rCBF Procedures}

Cerebral perfusion was assessed by using a commercial system (Novo Cerebrograph 32c) with 16 detectors covering each cerebral hemisphere. Reliability of detector placement across subjects was ensured by light markers aligned with the auditory meatus and orbitomeatal line. rCBF data acquisition was not blinded to clinical diagnosis, but the process is highly computerized and automated. It is extremely unlikely that the flow calculations or radioisotope counting could be biased by knowledge of diagnosis. Quality control procedures used have been previously described in detail. ${ }^{30}$ The rCBF measurements 
were performed for all subjects in a resting condition with eyes closed in a quiet and darkened room. Xenon133 was administered by inhalation for a period of $1 \mathrm{~min}-$ ute. The clearance curves were subsequently monitored for a total of 11 minutes. A six-parameter model (M2) was used to analyze the rCBF clearance curves to obtain values for initial slope index ${ }^{31}$ perfusion (ISI) and relative gray matter weight. ${ }^{22}$ The M2 method has shown greater reliability than other models in low-flow conditions typically seen with dementia. ${ }^{32}$ Of the total 896 clearance curves obtained across all subjects, 10 observations (1.1\%) in 7 cortical regions yielded monoexponential solutions and were treated as missing data for the wg analyses. As could be expected, 8 of these 10 monoexponential solutions occurred in frontal regions of the 2 patients in the FLD group who had the lowest frontal flows. The remaining two monoexponential values were observed in the frontotemporal regions of 2 patients in the depressed group.

\section{Statistical Analysis}

Group differences in demographic and clinical variables were assessed with analysis of variance (ANOVA) and chi-square tests where appropriate. Analysis of covariance (ANCOVA) was used to investigate differences in the global mean values for ISI and wg while controlling for the effects of $\mathrm{PCO}_{2}$. After statistically removing the effects of $\mathrm{PCO}_{2}$ with regression analyses, we performed pairwise group comparisons for the $\mathrm{PCO}_{2}$-adjusted global mean values by using the Newman-Keuls procedure. This multiple-range test maintains an overall significance level of $P<0.05$ for pairwise comparisons following a significant ANOVA. Regional detector values were normalized to the whole-cortex mean for both rCBF pa- rameters (ISI, wg). Repeated-measures ANOVAs were initially performed to analyze group effects for the regional detector values with hemisphere (right vs. left) as a within-subject factor. Because no significant groupby-hemisphere interactions were observed, regional values were bilaterally averaged across homologous regions of both hemispheres to simplify the analyses. The Newman-Keuls procedure was used for all subsequent pairwise comparisons, with a $P<0.05$ significance level. Pearson product-moment correlations were performed to assess the relationship between ISI and wg for each group.

\section{RESULTS}

Table 1 summarizes the demographic and clinical characteristics for the diagnostic groups. The groups did not differ significantly on distributions of age, years of education, and gender. In addition, the two dementia groups did not differ on a measure of dementia severity (mMMS) or illness duration, but there was a significant overall group effect for mMMS $(F=18.65, \mathrm{df}=3,24, P<0.0001)$, with both dementia groups scoring lower than the depressed patients and normal subjects, as expected. There was no significant difference between the depressed and normal groups on the mMMS. The four groups did not differ on end-tidal $\mathrm{PCO}_{2}$ or diastolic blood pressure levels assessed during $\mathrm{rCBF}$ measurement, but there was a significant difference for systolic blood pressure $(F=$ $3.88, \mathrm{df}=3,24, P<0.02$ ), with the depressed patients having lower systolic levels than the FLD group.

TABLE 1. Subject characteristics

\begin{tabular}{|c|c|c|c|c|}
\hline \multirow[b]{2}{*}{ Variable } & \multicolumn{4}{|c|}{ Mean \pm SD } \\
\hline & $\begin{array}{c}\text { FLD } \\
(n=7)\end{array}$ & $\begin{array}{c}\text { AD } \\
(n=7)\end{array}$ & $\begin{array}{c}\text { Depressed } \\
(n=7)\end{array}$ & $\begin{array}{c}\text { Normal } \\
(n=7)\end{array}$ \\
\hline Education (years) & $14.0 \pm 3.3$ & $14.0 \pm 3.6$ & $13.9 \pm 2.1$ & $14.0 \pm 2.3$ \\
\hline Gender $(\mathbf{M}, \mathbf{F})$ & 3,4 & 3,4 & 3,4 & 3,4 \\
\hline mMMS & $33.7 \pm 9.2^{\mathrm{a}}$ & $33.1 \pm 7.8^{\mathrm{a}}$ & $48.3 \pm 5.3$ & $54.7 \pm 0.5$ \\
\hline BP sys (mm Hg) & $144.9 \pm 13.4$ & $137.4 \pm 15.8$ & $122.1 \pm 10.7^{b}$ & $127.1 \pm 14.5$ \\
\hline BP dia (mm Hg) & $83.7 \pm 10.1$ & $83.7 \pm 15.5$ & $77.9 \pm 7.6$ & $76.9 \pm 7.4$ \\
\hline$P_{\mathrm{CO} 2}(\mathrm{~mm} \mathrm{Hg})$ & $36.1 \pm 2.2$ & $36.8 \pm 4.9$ & $38.3 \pm 5.0$ & $37.5 \pm 2.5$ \\
\hline \multicolumn{5}{|c|}{$\begin{array}{l}\text { Note: The Newman-Keuls procedure was used for multiple pairwise comparisons on variables that differed significantly by analysis of } \\
\text { variance. FLD = frontal lobe dementia; } \mathrm{AD}=\text { Alzheimer's disease; } \mathrm{M}=\text { male; } \mathrm{F}=\text { female; } \mathrm{mMMS}=\text { modified Mini-Mental State Examination } \\
\text { BP sys = systolic blood pressure; } \mathrm{BP} \text { dia }=\text { diastolic blood pressure; } P \mathrm{CO}_{2}=\text { partial pressure carbon dioxide. } \\
{ }^{\mathrm{a}} \text { Differs significantly from the depressed and normal groups }(P=0.0001) \text {. } \\
{ }^{b} \text { Differs significantly from the FLD group }(P=0.02) \text {. }\end{array}$} \\
\hline
\end{tabular}


Group Differences in Cortical Perfusion

The groups differed significantly in mean ISI, with or without controlling for the effects of $\mathrm{PCO}_{2}$ (Table 2). All three patient samples had lower mean perfusion than the normal subjects but did not differ significantly from each other. Regionally, as detailed in Table 2, the groups differed significantly in 10 of 16 cortical areas. Pairwise comparisons indicated significantly lower flows for the FLD group in frontal regions $\left(F_{3}, F_{4}\right)$ and relatively higher flows in temporal $\left(\mathrm{T}_{2}\right)$ and parieto-occipital $\left(\mathrm{P}_{4}, \mathrm{O}_{1}\right)$ regions compared with the other three groups. The FLD patients also showed lower flows than the $A D$ and depressed groups on an additional frontal detector $\left(F_{5}\right)$ and relatively higher flows in the parietotemporal $\left(\mathrm{P}_{1}\right)$ and occipital $\left(\mathrm{O}_{2}\right)$ regions compared with the depressed patients and normal control subjects, respectively. Figure 1 depicts the specific regional patterns of cortical perfusion in all four groups.

We also assessed differences between groups on an index of parietal deficit (PI index), defined as perfusion in the inferior parietal region $\left(\mathrm{P}_{1}, \mathrm{P}_{3}\right)$ referenced to the perirolandic $\left(\mathrm{C}_{1}\right)$ and occipital pole $\left(\mathrm{O}_{2}\right)$ regions. This index has previously shown sensitivity and specificity in discriminating $\mathrm{AD}$ from other demented and normal groups ${ }^{29,33}$ and is associated with decrements of cognitive performance in $\mathrm{AD} .^{34}$ The groups differed significantly on this parietal index (Table 2), with AD patients having a lower PI index score than the normal subjects; there were no other significant differences among the groups on the PI index.

To further assess group differences in anterior cortical flow, we computed an index of frontal lobe perfusion (FL index), defined as the ratio of mean perfusion in the anterior cortex $\left(F_{1}-F_{5}\right.$ detector regions) to mean flow in the posterior parietal $\left(\mathrm{P}_{4}\right)$ and occipital pole $\left(\mathrm{O}_{2}\right)$ regions. A significant group effect was observed for the FL index (see Table 2). Pairwise comparisons using the NewmanKeuls procedure indicated that the FLD group had mark-

TABLE 2. Summary of group differences in cortical perfusion

\begin{tabular}{|c|c|c|c|c|c|c|}
\hline \multirow[b]{2}{*}{ Variable } & \multicolumn{4}{|c|}{ Mean \pm SD } & \multirow[b]{2}{*}{$\boldsymbol{F}$} & \multirow[b]{2}{*}{$\boldsymbol{P}$} \\
\hline & $\begin{array}{c}\text { FLD } \\
(n=7)\end{array}$ & $\begin{array}{c}\text { AD } \\
(n=7)\end{array}$ & $\begin{array}{c}\text { Depressed } \\
(n=7)\end{array}$ & $\begin{array}{c}\text { Normal } \\
(n=7)\end{array}$ & & \\
\hline Global ISI & $38.6 \pm 7.6^{\mathrm{a}}$ & $39.0 \pm 4.3^{\mathrm{a}}$ & $43.0 \pm 5.2^{\mathrm{a}}$ & $49.3 \pm 7.5$ & 4.09 & 0.02 \\
\hline$F_{1}$ & $92.0 \pm 10.2$ & $102.3 \pm 5.8$ & $99.4 \pm 9.2$ & $104.2 \pm 10.0$ & 2.49 & 0.08 \\
\hline$F_{2}$ & $97.1 \pm 9.0$ & $101.2 \pm 7.3$ & $101.3 \pm 5.7$ & $105.3 \pm 3.2$ & 1.76 & NS \\
\hline$F_{3}$ & $93.2 \pm 9.5^{b}$ & $104.7 \pm 3.0$ & $101.1 \pm 5.1$ & $100.9 \pm 2.6$ & 4.95 & 0.01 \\
\hline$F_{4}$ & $92.2 \pm 7.1^{b}$ & $102.7 \pm 4.6$ & $99.1 \pm 3.8$ & $105.0 \pm 4.7$ & 8.03 & 0.001 \\
\hline$F_{5}$ & $96.8 \pm 5.9$ & $107.5 \pm 4.4^{\mathrm{a}, \mathrm{c}}$ & $104.3 \pm 2.9^{c}$ & $101.3 \pm 3.2$ & 8.02 & 0.001 \\
\hline $\mathrm{C}_{1}$ & $102.7 \pm 5.3$ & $101.7 \pm 6.2$ & $101.9 \pm 3.8$ & $100.9 \pm 3.5$ & 0.16 & NS \\
\hline $\mathrm{C}_{2}$ & $103.5 \pm 3.8$ & $108.0 \pm 6.0$ & $101.7 \pm 4.5$ & $102.5 \pm 5.8$ & 2.17 & NS \\
\hline$T_{1}$ & $90.7 \pm 7.0$ & $99.0 \pm 3.7$ & $93.6 \pm 10.3$ & $94.4 \pm 5.9$ & 1.14 & NS \\
\hline$T_{2}$ & $107.6 \pm 6.0^{b}$ & $101.0 \pm 4.8$ & $100.4 \pm 5.5$ & $98.4 \pm 2.7$ & 4.73 & 0.01 \\
\hline$T_{3}$ & $102.7 \pm 3.8^{\mathrm{a}}$ & $96.6 \pm 1.6^{c}$ & $103.9 \pm 7.4^{\mathrm{a}, \mathrm{d}}$ & $96.0 \pm 1.5$ & 6.26 & 0.003 \\
\hline$P_{1}$ & $103.1 \pm 3.5$ & $91.2 \pm 3.5^{b}$ & $98.2 \pm 3.9^{c}$ & $99.5 \pm 3.0$ & 14.17 & 0.0001 \\
\hline$P_{2}$ & $102.0 \pm 5.1$ & $94.5 \pm 4.5^{c}$ & $97.4 \pm 4.0$ & $96.6 \pm 3.2$ & 3.98 & 0.02 \\
\hline$P_{3}$ & $97.5 \pm 3.9$ & $92.6 \pm 5.8$ & $94.2 \pm 5.5$ & $98.1 \pm 3.5$ & 2.15 & NS \\
\hline $\mathbf{P}_{4}$ & $108.1 \pm 5.0^{b}$ & $101.5 \pm 5.1$ & $101.5 \pm 3.5$ & $101.2 \pm 3.4$ & 4.20 & 0.02 \\
\hline $\mathrm{O}_{1}$ & $106.3 \pm 7.7^{b}$ & $96.1 \pm 4.9$ & $100.5 \pm 4.5$ & $98.0 \pm 2.8$ & 5.04 & 0.01 \\
\hline $\mathrm{O}_{2}$ & $104.3 \pm 3.9^{\mathrm{a}}$ & $99.5 \pm 3.3$ & $101.7 \pm 5.5$ & $97.6 \pm 3.8$ & 3.32 & 0.04 \\
\hline PI Index & $97.0 \pm 3.2$ & $91.5 \pm 5.6^{\mathrm{a}}$ & $94.6 \pm 5.9$ & $99.6 \pm 2.4$ & 4.06 & 0.02 \\
\hline FL Index & $88.9 \pm 7.1^{b}$ & $103.2 \pm 3.9$ & $99.7 \pm 7.0$ & $104.1 \pm 4.9$ & 9.72 & 0.0002 \\
\hline
\end{tabular}

Note: Analysis of covariance was used for overall group differences in global perfusion (initial slope index units) with $\mathrm{PCO}_{2}$ as a covariate. Analysis of variance was used for regional values normalized to global mean ISI. The Newman-Keuls procedure was used for pairwise comparisons on regional and $\mathrm{PCO}_{2}$-adjusted global mean values. $\mathrm{FLD}=$ frontal lobe dementia; $A D=$ Alzheimer's disease; $\mathrm{F}_{1}-\mathrm{F}_{5}=$ detectors in the frontal regions; $C_{1}$ and $C_{2}=$ detectors in the perirolandic regions; $T_{1}-T_{3}=$ detectors in the temporal regions; $P_{1}-P_{4}=$ detectors in the parietal regions; $\mathrm{O}_{1}$ and $\mathrm{O}_{2}=$ detectors in the occipital regions; $\mathrm{PI}$ Index $=$ flow in the parietotemporal region $\left(\mathrm{P}_{1}, \mathrm{P}_{3}\right)$ referenced to the perirolandic $\left(C_{1}\right)$ and occipital $\left(\mathrm{O}_{2}\right)$ regions; $\mathrm{FL}$ Index $=$ flow in the anterior cortex $\left(\mathrm{F}_{1}-\mathrm{F}_{5}\right.$ detectors) referenced to parieto-occipital $\left(\mathrm{P}_{4}, \mathrm{O}_{2}\right)$ flow; $N S=$ not significant. ${ }^{2}$ Differs from normal control subjects.

biffers from the other three groups.

'Differs from the FLD group.

${ }^{d}$ Differs from the AD group. 
edly lower FL index scores than the AD, depressed, and normal groups. There were no other significant differences among the groups on the FL index. Similarly, and for comparison with other published data, we computed the conventional hyperfrontality index as the five frontal regions divided by the 11 other areas (anterior/posterior). The values of this index were $92.0 \pm 6.8,105.5 \pm 4.4$, $101.5 \pm 4.4$, and $105.0 \pm 3.6$ in the FLD, AD, MDD, and normal subjects, respectively $(F=11.24, \mathrm{df}==24, P<$ 0.0001).

\section{Group Differences in Gray Matter Weight}

The groups differed significantly on global mean wg, with or without controlling for the effects of $\mathrm{PCO}_{2}$ (Table 3). The FLD group had significantly lower global wg than the MDD and normal groups; there were no other significant differences. Regionally, the groups differed significantly in five detectors involving the frontal $\left(\mathrm{F}_{4}\right)$, temporal $\left(T_{1}\right)$, parietal $\left(P_{4}\right)$, and occipital $\left(\mathrm{O}_{2}\right)$ regions (Table 3). The FLD group had relatively lower wg values in the frontal $\left(\mathrm{F}_{4}\right)$ and anterior temporal $\left(\mathrm{T}_{1}\right)$ regions than the $A D$ patients and lower $\mathrm{wg}$ in the frontal region $\left(\mathrm{F}_{4}\right)$ than the depressed patients. Further, the FLD group had relatively higher $\mathrm{wg}$ in the posterior parietal $\left(\mathrm{P}_{4}\right)$ and occipital pole $\left(\mathrm{O}_{2}\right)$ regions than the other three groups. No other pairwise group differences were observed.

Regional patterns of $\mathrm{wg}$ for the groups are depicted in Figure 2. To specifically assess group differences in wg for parietotemporal and frontal regions, we compared groups on the PI and FL index scores for wg values. There was no significant group effect for the PI index, but the groups differed significantly on the FL index for wg (Table 3): the FLD group had lower FL index scores than each of the other three groups.

FIGURE 1. Comparison of regional patterns of perfusion (initial slope index units) for the FLD, AD, depressed, and normal groups. Regional detector values of cortical perfusion are expressed as percentages of whole-cortex mean flow and averaged across right and left hemispheres. The red end of the color scale indicates relatively higher perfusion values; the blue end of the scale represents lower flows. Note the relative reductions of perfusion in the parietotemporal region for the AD patients contrasted to the marked reductions of flow in the frontotemporal regions of the FLD group. FLD = frontal lobe dementia; AD = Alzheimer's disease.

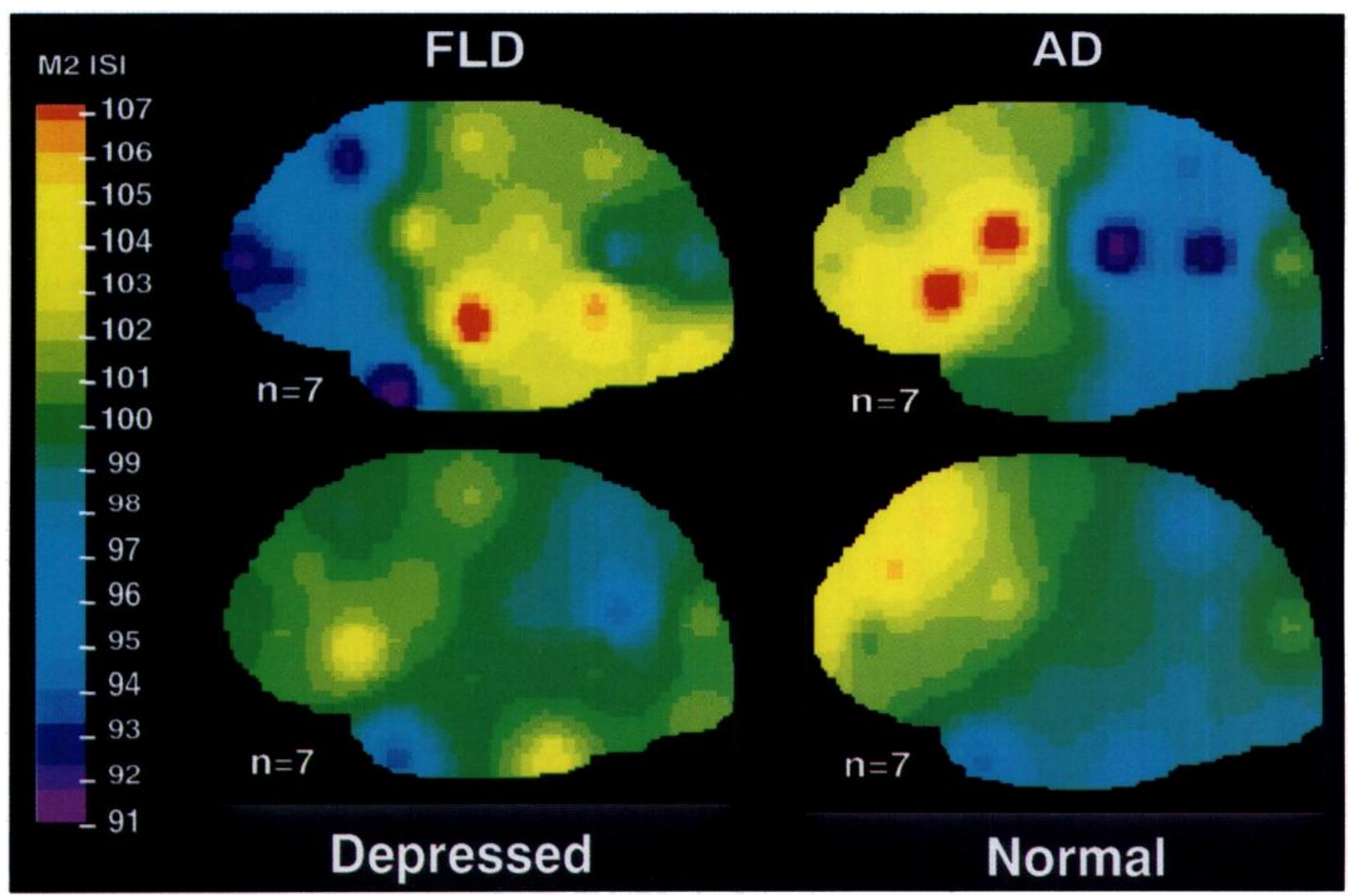


TABLE 3. Summary of group differences in relative gray matter weight (wg)

\begin{tabular}{|c|c|c|c|c|c|c|}
\hline \multirow[b]{2}{*}{ Variable } & \multicolumn{4}{|c|}{ Mean \pm SD } & \multirow[b]{2}{*}{$\boldsymbol{F}$} & \multirow[b]{2}{*}{$\boldsymbol{P}$} \\
\hline & $\begin{array}{c}\text { FLD } \\
(n=7)\end{array}$ & $\begin{array}{c}\text { AD } \\
(n=7)\end{array}$ & $\begin{array}{c}\text { Depressed } \\
(n=7) \\
\end{array}$ & $\begin{array}{c}\text { Normal } \\
(n=7)\end{array}$ & & \\
\hline$F_{1}$ & $88.7 \pm 7.9(n=6)$ & $98.4 \pm 4.8$ & $93.3 \pm 10.1$ & $99.4 \pm 5.3$ & 2.94 & 0.055 \\
\hline $\mathrm{F}_{4}$ & $85.7 \pm 2.5$ & $93.3 \pm 4.1^{b}$ & $93.9 \pm 4.2^{c}$ & $88.6 \pm 5.0$ & 6.51 & 0.002 \\
\hline $\mathrm{T}_{1}$ & $85.1 \pm 13.4$ & $101.4 \pm 7.5^{b}$ & $91.3 \pm 10.8$ & $99.5 \pm 8.9$ & 3.45 & 0.03 \\
\hline $\mathrm{O}_{2}$ & $116.0 \pm 6.5^{c}$ & $107.8 \pm 6.2$ & $107.2 \pm 1.7$ & $102.8 \pm 3.6$ & 8.91 & 0.0004 \\
\hline FL Index & $80.6 \pm 6.4^{c}$ & $92.2 \pm 4.8$ & $91.3 \pm 4.3$ & $94.2 \pm 4.2$ & 10.45 & 0.0001 \\
\hline
\end{tabular}

Analysis of covariance was used for overall group differences in global wg, with $\mathrm{PCO}_{2}$ as a covariate. Analysis of variance was performed for regional values normalized to global mean wg. The Newman-Keuls procedure was used for multiple pairwise comparisons in regional and $\mathrm{PCO}_{2}$-adjusted global mean values. One FLD patient had a monoexponential solution at the $\mathrm{F}_{1}$ detector region; this was treated as missing data. $F L D=$ frontal lobe dementia; $A D=$ Alzheimer's disease; $F_{1}$ and $F_{4}=$ percent gray matter weight for detectors in frontal regions; $T_{1}=$ percent gray matter weight for a temporal detector region; $P_{4}=$ percent gray matter weight for a parietal detector region; $\mathrm{O}_{2}=$ percent gray matter weight for an occipital detector region; FL Index $=$ percent gray-matter weight in the anterior cortex $\pm F_{1}-F_{5}$ detectors referenced to gray matter weight in the parieto-occipital $\pm \mathrm{P}_{4}, \mathrm{O}_{2}$ regions.

${ }^{a}$ Differs from the normal control group.

Differs from the FLD group.

'Differs from the other three groups.

Finally, we assessed the relationship between ISI and wg by examining correlations for each group between these two rCBF parameters. Although the sample sizes were small, a significant positive correlation was observed in the FLD group between the mean cortical ISI and $\mathrm{wg}$ values $(r=0.76, \mathrm{df}=6, P<0.05)$. No significant association between the global mean values of ISI and wg were found in the other groups, and there were no significant correlations between ISI and wg for the PI and FL indices in any of the four groups.

\section{DISCUSSION}

Our sample of FLD patients showed relatively lower flows in the frontal cortical regions than the $A D$, depressed, and normal groups, consistent with findings in previous studies. The magnitude of this frontal CBF deficit was further demonstrated by the markedly lower scores of the FLD group on an index of frontal perfusion, which assessed perfusion in the frontal cortex relative to specific posterior parietal and occipital pole regions or all posterior areas. On our index of parietal deficit, the AD patients differed significantly from normal subjects; however, the differences from the FLD and depressed patients did not reach significance, highlighting the limitation of this simple parietal index when applied to single cases or small groups of patients. Global mean CBF was also lower in the $A D, F L D$, and depressed patients than the normal subjects. Although MDD patients had higher flow than the two dementia groups, this difference failed to reach significance with these small samples. That the FLD patients showed a regional flow deficit that was clearly distinguishable from the other groups, despite the small sample size, supports the use of functional imaging in the clinical diagnosis of FLD and highlights the importance of considering regional differences in perfusion among patient groups.

Although previous studies have shown reductions of cortical CBF in FLD, regional patterns of the gray matter weight index have not been previously reported among patients with this type of dementia. We found that the FLD patients had significantly lower wg values in the frontotemporal regions than the AD and depressed patients. Specifically, they showed markedly lower scores on the frontal index for wg compared with each of the three comparison groups. This difference suggests a focal loss of gray matter in the frontal cortex for the FLD patients that was not observed in the other groups. The relatively higher wg values for the FLD patients in the posterior parietal and occipital cortices suggests that these regions are relatively unaffected for these patients.

The validity of such changes, for both ISI and wg, is being investigated in an autopsy series. Initial findings suggest excellent validity of $\mathrm{rCBF}$ findings against subsequent neuropathological examination. ${ }^{35}$ Effects for global wg values appeared less robust in discriminating the FLD patients from the comparison groups. The FLD patients had significantly lower global wg values than the depressed patients and normal control subjects; they did not, however, differ from the $\mathrm{AD}$ patients. 

FIGURE 2. Comparison of regional patterns of gray matter weight (wg) for the FLD, AD, depressed, and normal groups. Regional detector values of gray matter weight are expressed relative to whole-cortex mean $\mathrm{wg}$ and averaged across right and left hemispheres. The red end of the color scale indicates relatively higher wg values; the blue end of the scale represents lower weight scores. Note the marked reductions of gray matter weight in the frontotemporal cortex for the FLD patients compared with the other groups. FLD = frontal lobe dementia; AD = Alzheimer's disease.

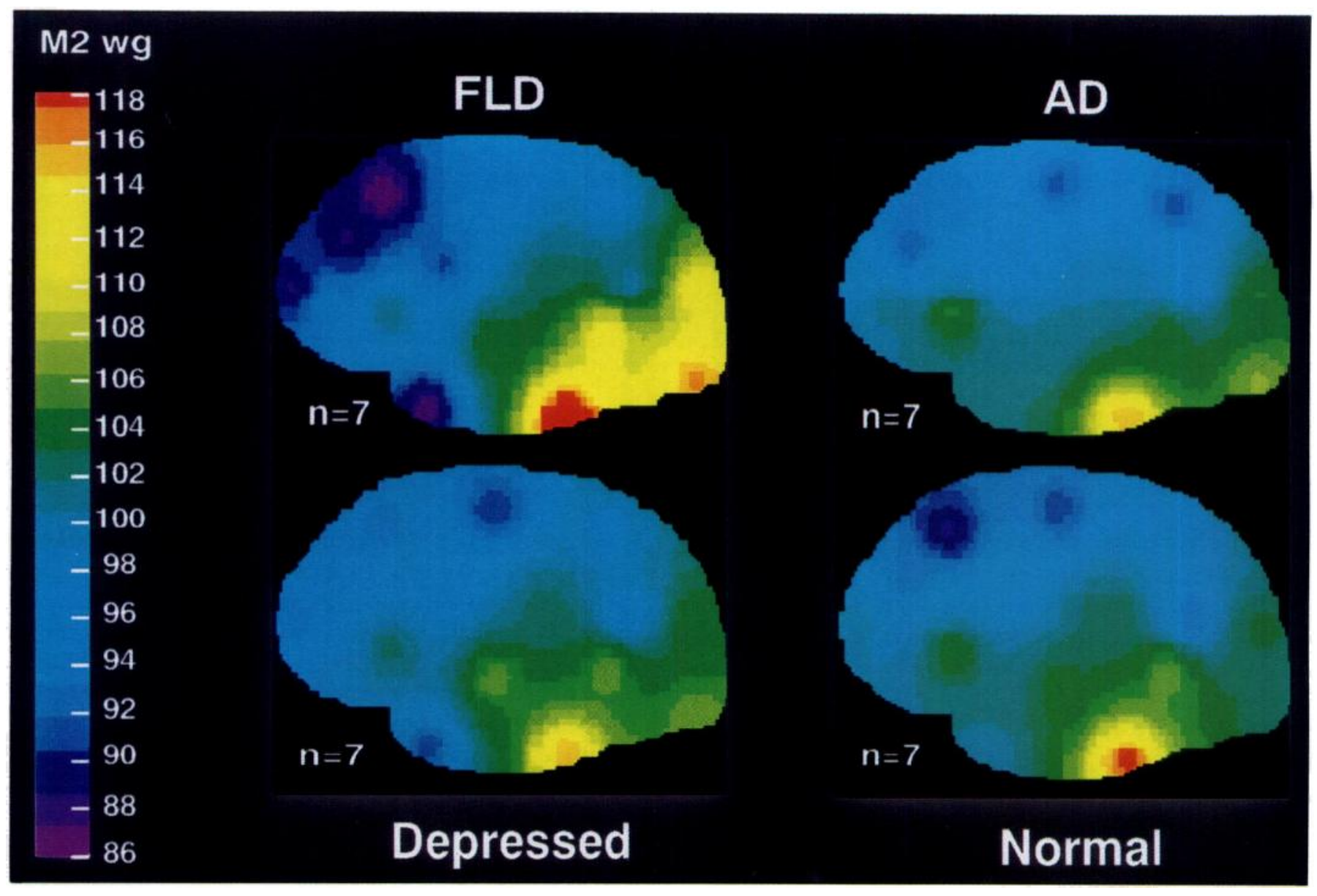

Although global values of perfusion and gray matter weight differed between groups, the most prominent between-group differences were in regional patterns on these $\mathrm{rCBF}$ variables. Hypofrontality of $\mathrm{CBF}$ and metabolism among severely depressed patients has been shown in several studies. ${ }^{17-21}$ The deficits in perfusion and gray matter weight in the anterior cortex of the FLD patients were clearly distinguishable from the effects of severe depression in this geriatric sample. Although one FLD patient was taking psychotropic medication at the time of rCBF measurement, it is unlikely that the prominent regional deficits observed in our FLD sample could be explained by a medication effect. In fact, the differences between the FLD patients and the three comparison groups remained significant for $\mathrm{CBF}$ and $\mathrm{wg}$ on the FL index after excluding the medicated patient from the analyses.
It is also important to note that 2 FLD patients had monoexponential solutions for several detectors assessing $w g$ in the frontal cortex. Examination of the ISI values for these detectors suggests that gray matter flow was very low in the anterior cortex for these 2 patients and probably indistinguishable from white matter perfusion, accounting for the monoexponential solutions in this cortical region. Although the monoexponential solutions were treated as missing data in our wg analyses, their occurrence in the frontal region for the 2 FLD patients is consistent with the regional deficit in gray matter weight observed in the remainder of the FLD sample.

The FLD patients showed marked reductions in the anterior cortex for both CBF and wg. This finding suggests a strong correspondence between regional deficits in cortical perfusion and gray matter weight in FLD that is not present in $\mathrm{AD}$ and severe depression. The signifi- 
cant correlation observed between global mean perfusion and wg in only the FLD group further supports this finding. Given that xenon-133 is inert and freely diffusible, the differences observed between groups on wg, suggesting greater cortical cell loss in FLD than in the other groups, would not substantially confound the measurement of perfusion with ISI. Thus, the perfusion deficit that has been reported among FLD patients may represent a different neurophysiological abnormality than has been observed in AD or severe depression. Specifically, the association between the $\mathrm{rCBF}$ indices of perfusion and gray matter weight may reflect $C B F$ deficits that are related to greater cortical cell loss or atrophy than is typically observed among $\mathrm{AD}$ patients with similar levels of dementia severity. In fact, the regional pattern of wg for the AD group was not appreciably different from those for our depressed and normal groups.

The two dementia groups performed at similar levels on a screening measure of cognitive functioning. However, in contrast to the AD group, which showed perfusion deficits in the parietotemporal region, the FLD patients showed deficits in both regional perfusion and gray matter weight in the anterior cortex, suggestive of greater neuropathological severity in that region. This explanation suggests that, for a given level of overall cognitive function, the disease process of FLD may produce greater neuronal cell loss in the affected brain region (frontal cortex) than in the parietal region of $A D$ and that the physiological effects of $A D$ may be associated with a compromise of neuronal function without the same degree of cell loss observed in FLD. An alternative explanation for this finding may be that the cognitive screening measure of dementia severity used to match the AD and FLD groups (the modified Mini-Mental State Examination) is less sensitive to the effects of frontal lobe dysfunction than to the effects of $A D$. This would suggest that the FLD patients required a more severe dementia to reach the same level of performance on this measure of cognitive functioning as the AD patients. The two dementia groups did not differ on estimates of dementia duration. Thus, the distinguishing pathophysiological findings observed in the FLD group may reflect a faster progression of the physiological effects of FLD than occurs in $A D$ and may be attributed to very different neurodegenerative processes whose etiologies have yet to be discerned. Longitudinal studies of $\mathrm{CCBF}$ in FLD are needed to address these questions more directly.

Our findings provide further evidence that functional imaging has clinical utility in the differential diagnosis of FLD. Using $\mathrm{rCBF}$ indices of cortical perfusion and gray matter weight, we report deficits in both $\mathrm{rCBF}$ variables involving the anterior cortex that distinguished the effects of FLD from the effects of AD, severe geriatric depression, and normal aging. Further, we observed a significant association between global mean perfusion and wg values among FLD patients that was not found in the other groups. This correspondence between deficits in perfusion and gray matter weight in FLD suggests different pathophysiological effects in FLD than in AD, despite similar durations of illness and performance on a measure of cognitive impairment. Together, these findings point to a different neurodegenerative process in FLD, with pathophysiological effects that can be distinguished from those typically observed in $A D$, depression, and normal aging.

This work was supported by grants from the National Institute on Aging (AG07370, AG07232, AG05433, AG10638, AG08702), the National Institute of Mental Health (MH35636, MH44779), the Charles S. Robertson Memorial Gift for Alzheimer's Disease, and the Jean and Louis Dreyfus Foundation. An early version of this paper was presented at the meeting of the American Psychiatric Association in Washington, DC, May 2-7, 1992.

\section{References}

1. Gustafson L: Frontal lobe degeneration of non-Alzheimer's type, II: clinical picture and differential diagnosis. Archives of Gerontology and Geriatrics 1987; 6:209-223

2. Neary D, Snowden JS, Northen B, et al: Dementia of frontal lobe type. J Neurol Neurosurg Psychiatry 1988; 51:353-361

3. Munoz-Garcia D, Ludwin SK: Classic and generalized variants of Pick's disease: a clinicopathological, ultrastructural, and immunocytochemical comparative study. Ann Neurol 1984; 16:467-480

4. Brun A: Frontal lobe degeneration of non-Alzheimer's type, I: neuropathology. Arch Gerontol Geriatr 1987; 6:193-207

5. Graff-Radford NR, Damasio AR, Hyman BT, et al: Progressive aphasia in a patient with Pick's disease: a neuropsychological, radiologic, and anatomic study. Neurology 1990; 40:620-625

6. Knopman DS, Mastri AR, Frey WH, et al: Dementia lacking distinctive histologic features: a common non-Alzheimer degenerative dementia. Neurology 1990; 40:251-256

7. Snowden JS, Neary MD, Mann AMA, et al: Progressive language disorder due to lobar atrophy. Ann Neurol 1992; 31:174-183

8. Knopman DS, Christensen KJ, Schut LJ, et al: The spectrum of imaging and neuropsychological findings in Pick's disease. Neurology 1989; 39:362-368

9. Kuwabara $Y$, Ichiya $Y$, Otsuka M, et al: Comparison of I-123 IMP and Tc-99m HMPAO SPECT studies with PET in dementia. Ann Nucl Med 1990; 4:75-82

10. Neary D, Snowden JS: Dementia of the frontal lobe type, in Frontal Lobe Function and Dysfunction, edited by Levin HS, Eisenberg HM, Benton AL. New York, Oxford University Press, 1991, pp 304-317

11. Neary D, Snowden JS, Shields RA, et al: Single photon emission tomography using 99m-Tc HMPAO in the investigation of dementia. J Neurol Neurosurg Psychiatry 1987; 50:1101-1109

12. Risberg J: Frontal lobe degeneration of the non-Alzheimer's type, III: regional cerebral blood flow. Archives of Gerontology and Geriat- 


\section{FRONTAL LOBE DEMENTIA}

rics $1987 ; 6: 225-233$

13. Jagust WJ, Reed BR, Seab JP, et al: Clinical-physiologic correlates of Alzheimer's disease and frontal lobe dementia. Am J Physiol Imaging 1989; 4:89-96

14. Miller BL, Cummings JL, Villanueva-Meyer J, et al: Frontal lobe degeneration: clinical, neuropsychological, and SPECT characteristics. Neurology 1991; 41:1374-1382

15. Risberg J, Gustafson L: Regional cerebral blood flow in psychiatric disorders, in Handbook of Regional Cerebral Blood Flow, edited by Knezevic S, Maximillian VA, Mubrin Z, et al. Hillsdale, NJ, Lawrence Erlbaum, 1988, pp 219-240

16. Baxter LR Jr, Schwartz JM, Phelps ME, et al: Reduction of prefrontal cortex glucose metabolism common to three types of depression. Arch Gen Psychiatry 1989; 46:243-252

17. Sackeim HA, Prohovnik I, Moeller JR, et al: Regional cerebral blood flow in mood disorders, I: comparison of major depressives and normal controls at rest. Arch Gen Psychiatry 1990; 47:60-70

18. Guze BH, Baxter LR Jr, Schwartz JM, et al: Changes in glucose metabolism in dementia of the Alzheimer's type compared with depression: a preliminary report. Psychiatry Res: Neuroimaging 1991; 40:195-202

19. Dolan RJ, Bench CJ, Brown RG, et al: Regional cerebral blood flow abnormalities in depressed patients with cognitive impairment. J Neurol Neurosurg Psychiatry 1992; 55:768-773

20. Sackeim HA, Prohovnik I, Moeller JR, et al: Regional cerebral blood flow in mood disorders, II: comparison of major depression and Alzheimer's disease. J Nucl Med 1993; 34:1090-1101

21. Hansen LA, Deteresa R, Tobias H, et al: Neocortical morphometry and cholinergic neurochemistry in Pick's disease. Am J Pathol 1988; 131:507-518

22. Obrist WD, Wilkinson WE: The non-invasive Xe-133 method: evaluation of CBF indices, in Cerebral Circulation and Neurotransmitters, edited by Bes A, Geraud G. Amsterdam, Exerpta Medica, 1979, pp 119-124

23. Prohovnik I, Smith G, Sackeim H A, et al: Gray-matter degeneration in presenile Alzheimer's disease. Ann Neurol 1989; 25:117-124

24. McKhann G, Drachman D, Folstein M, et al: Clinical diagnosis of Alzheimer's disease: report of the NINCDS-ADRDA Work Group under the auspices of the Department of Health and Human Services Task Force on Alzheimer's disease. Neurology 1984; 34:939-944

25. Folstein MF, Folstein SE, McHugh PR: "Mini-Mental State": a practical method for grading the cognitive state of patients for the clinician. J Psychiatr Res 1975; 12:189-198

26. Mayeux R, Stern Y, Rosen J, et al: Depression, intellectual impairment and Parkinson disease. Neurology 1981; 31:645-650

27. Endicott J, Spitzer R: A diagnostic interview: the Schedule for Affective Disorders and Schizophrenia. Arch Gen Psychiatry 1978; 35:837-844

28. Hamilton M: Development of a rating scale for primary depressive illness. British Journal of Social and Clinical Psychology 1967; 6:278296

29. Prohovnik I, Mayeux R, Sackeim HA, et al: Cerebral perfusion as a diagnostic marker of early Alzheimer's disease. Neurology 1988; 38:931-937

30. Prohovnik I: Data quality, integrity, and interpretation, in Handbook of Regional Cerebral Blood Flow, edited by Knezevic S, Maximillian VA, Mubrin Z, et al. Hillsdale, NJ, Lawrence Erlbaum, 1988, pp 51-78

31. Prohovnik I, Knudsen E, Risberg J: Theoretical evaluation and simulation test of the initial slope index for noninvasive $\mathrm{CBF}$, in Cerebral Blood Flow and Metabolism Measurement, edited by Hartmann $\mathrm{H}$, Hoyer S. Berlin, Springer-Verlag, 1985, pp 56-60

32. Prohovnik I, Knudsen E, Risberg J: Accuracy of models and algorithms for determination of fast-compartment flow by non-invasive 133-Xe clearance, in Functional Radionuclide Imaging of the Brain, edited by Magistretti P. New York, Raven, 1983, pp 87-115

33. Prohovnik I, Alexander GE, Tatemichi TK, et al: Exploring the nature of the parietotemporal perfusion deficit in AD (abstract). J Cereb Blood Flow Metab 1991; 11(suppl 2):179

34. Alexander GE, Prohovnik I, Stern Y, et al: WAIS-R subtest profile and cortical perfusion in Alzheimer's disease. Brain Cognition 1994; 24:143-171

35. Dwork AJ, Prohovnik I: Regional cerebral blood flow and postmortem findings in lobar atrophy (abstract). J Neuropathol Exp Neurol 1992; 51:316 